ORIGINAL ARTICLE

\title{
SNP discovery, expression and association analysis for the SDHD gene in pigs
}

\author{
S.E.F. Guimaraes ${ }^{1} \star$, M.F. Rothschild ${ }^{1}$, D. Ciobanu ${ }^{2}$, C.H. Stahl ${ }^{1} \&$ S.M. Lonergan ${ }^{1}$ \\ 1 Department of Animal Science and Center for Integrated Animal Genomics, Iowa State University, Ames, IA, USA \\ 2 PIC USA, Franklin, KY, USA
}

\author{
Keywords \\ growth; normalized gene expression; pig \\ production; pork quality; semiquantitative real- \\ time PCR; succinate dehydrogenase complex. \\ Correspondence \\ Simone E. F. Guimaraes, Departmento de \\ Zootecnia, Universidade Federal de Vicosa, \\ Vicosa, Minas Gerais 36571-000, Brazil. Tel: \\ 55-031-3899-2273; Fax: 55-031-3899-2275; \\ E-mail: sfacioni@ufv.br \\ *Present address: \\ Animal Science Department, Federal University \\ of Vicosa, Vicosa, MG 36571-000, Brazil.
}

\begin{abstract}
Summary
The SDHD gene was examined for single nucleotide polymorphisms (SNP) as well as for expression changes in the Longissimus dorsi muscle of commercial pigs with different potential for growth. Three SNPs, including one previously described in the coding region and two new ones in the 3'-UTR, were found. The normalized expression of SDHD was correlated with growth, meat quality and sensory traits $(\mathrm{p}<0.05)$. For the commercial pigs used in this study, as well as a Berkshir$\mathrm{e} \times$ Yorkshire resource population, the SNPs have been associated $(\mathrm{p}<0.05)$ with: growth, carcass composition, meat quality and sensory traits. Despite the fact that the described SNPs were not significantly associated with the normalized expression values, the SDHD SNPs and expression were associated with growth and meat quality traits in pigs.
\end{abstract}

Received: 13 October 2006;

accepted: 15 February 2007

\section{Introduction}

Genes within pathways related to energy metabolism make attractive targets for single nucleotide polymorphism (SNP) discovery and association studies to identify markers associated with increased growth performance and pigs. Succinate-ubiquinone oxidoreductase (cytochrome $b$ ) is an important enzyme complex in both the tricarboxylic acid cycle and the electron transport system required for aerobic respiration in both eukaryotic cells and prokaryotic organisms. SDHD (succinate dehydrogenase complex, subunit $\mathrm{D}$ ) is one of the four subunits of succinate dehydrogenase complex (SHDB, SDHC, SDHD and fumarate hydratase), and in humans, mutations in these genes have been involved in familial predisposition to benign tumours (Gottlieb \& Tomlinson 2005).

Previous work in cattle has shown biallelic expression of $S D H D$ in a wide range of fetal and adult tissues (Khatib 2005). Zhu et al. (2005) obtained a cDNA clone of $S D H D$ from a Chinese Tongcheng pig 55-day fetal Longissimus dorsi muscle cDNA library, and by using the INRA-University of Minnesota porcine radiation hybrid panel determined its map position close to marker SW2401 on SSC9. After SNP and association analysis, SDHD genotypes were significantly $(\mathrm{p}<0.05)$ associated with average loin muscle area. No other authors have discussed previously the association of SDHD with growth, carcass and meat quality traits. In the present study, we considered the potential of $S D H D$ as a candidate gene for production traits due to its key role in the $S D H D$ complex, in the process of aerobic respiration. The $S D H D$ cDNA sequence for new SNPs, the expression levels of $S D H D$ mRNA in the Longissimus dorsi muscle of a commercial pig population, and their association with pig growth performance, carcass composition and meat quality traits were examined.

\section{Material and methods}

Animals

A commercial pig population consisting of 120 animals was derived from the cross between a commercial line 
of Duroc sires and dams from a synthetic white line. The pigs were selected from two groups formed according to the sires' estimated breeding value (EBV) for growth. These two growth groups included the fast-growing animals (EBVF) and the slow-growing animals (EBVS) based on sire's EBV for age to $125 \mathrm{~kg}$ (AGE125). Table 1 summarizes some of the phenotypic differences between both groups. In addition, 24 animals from each breed (total of 96) Landrace, Pietrain, Yorkshire and Duroc were tested for the genotypic and allele frequencies.

\section{RNA isolation and cDNA synthesis}

A sample of the Longissimus dorsi muscle was collected after slaughter for all the animals, treated with RNAlater (Ambion, Austin, TX, USA) and stored until RNA isolation. Total RNA was extracted using the RNeasy Midi Kit (Qiagen, Valencia, CA, USA), and the DNAse I treatment was made using the on-column DNase digestion during RNA purification, using the Qiagen RNase-free DNase - set (Qiagen). The purified RNA was reverse transcribed with Superscript III Kit (Invitrogen Life Technologies, Carlsbad, CA, USA) according to the manufacturer's instructions. The cDNA was then incubated for $20 \mathrm{~min}$ at $37^{\circ} \mathrm{C}$ with Escherichia coli RNase $\mathrm{H}$ and stored at $-80^{\circ} \mathrm{C}$ until analysis by semiquantitative real-time PCR. cDNA samples were tested for genomic DNA contamination by regular PCR using different primer sets available in the laboratory, and no amplification was seen in $1.5 \%$ agarose gels.

\section{Primer design and PCR optimization}

A pair of primers was designed to amplify $845 \mathrm{bp}$ of the SDHD cDNA, based on the EST sequence TC219190 (http://www.tigr.org) using the software PRIMER3, available at http://frodo.wi.mit.edu/cgi-bin/ primer3/primer3_www.cgi. Primer sequences, reaction components and thermocycling conditions are summarized in Table 2.

\section{SNP identification}

For sequencing and SNP identification, the SDHD cDNA amplicon was sequenced using the five most extreme animals in each growth group (EBVF and EBVS). Three SNPs were identified in the cDNA: G444T (previously described by Zhu et al. 2005), T859C and G860A. Primers for the three SNPs were designed using 'PRIMERQUEST' software available from Integrated DNA Technologies, Inc. (Coralville, IA,
USA) and PCR-RFLP tests were developed for all SNPs (Table 2).

Linkage mapping of the SDHD gene in pigs and QTL analysis

A Berkshire $\times$ Yorkshire $\mathrm{F}_{2}(\mathrm{BY})$ population described in Malek et al. (200la,b) was used for the linkage mapping of SDHD. The G444T SNP and CRIMAP software version 2.4 (Green et al. 1990) were used to map this gene among the 12 microsatellite markers described by Malek et al. (200la,b). To verify if the QTL profile on SSC9 described by Malek et al. $(200 \mathrm{la}, \mathrm{b})$ was changed by the inclusion of SDHD gene mapping, a QTL analysis was conducted using the QTLEXPRESS software (Seaton et al. 2002), found in the site http://qtl.cap.ed.ac.uk/. The sex-average linkage map was used in the QTL analysis.

\section{Semiquantitative real-time PCR}

To quantify transcript levels of $S D H D$, semiquantitative real-time PCR was performed using the $\mathrm{MyiQ}^{\mathrm{TM}}$ Single Color Real-Time PCR Detection System (BioRad Laboratories, Hercules, CA, USA). The primers used for the reactions were the same described for the G860A SNP genotyping. Reactions were performed in a final volume of $25 \mu \mathrm{l}$ using $12.5 \mu \mathrm{l}$ of $2 \mathrm{X}$ SYBR Green Supermix (Bio-Rad), $400 \mathrm{~nm}$ of each primer and $100 \mathrm{ng}$ of the above-described cDNA. Prior to quantification by semiquantitative real-time PCR, optimal primer concentrations were determined. It was verified that $S D H D$ amplification was linear and similar to that of the control gene, 60S ribosomal RNA (RPL35), that had a not different amplification pattern between both EBV groups $(p>0.05)$. The thermal cycling conditions allowed for 45 cycles of $30 \mathrm{~s}$ of melting at $95^{\circ} \mathrm{C}$ followed by $30 \mathrm{~s}$ of annealing and extension at $60^{\circ} \mathrm{C}$. After the 45 amplification cycles, all samples were subjected to a melt curve analysis in which they were heated at $1{ }^{\circ} \mathrm{C}$ increments from 60 to $94^{\circ} \mathrm{C}$ to validate the absence of non-specific products. Normalized gene expression is presented using the 24Ct method (Livak \& Schmittgen 2001).

\section{Statistical analysis}

Associations between the genotypes, the normalized expression values $\left(2^{-\Delta \mathrm{Ct}}\right)$ and the phenotypic traits were tested using the general linear model procedure (sAs procedure GLM, SAS Institute, Cary, NC, USA) with a model that included EBV group, genotype, sex, sire within EBV group, dam within sire and 
EBV group, interaction between EBV group and genotype. For the meat quality and sensory traits, slaughter day was also included in the model. Least square mean values for the genotypes and for the interaction between EBV groups and genotypes were obtained. The comparison between EBVF and EBVS $S D H D$ gene expression averages was carried out by $t$ test. Pearson correlations were used to calculate the correlations between $2^{-4 \mathrm{Ct}}$ values and phenotypic traits. Association analysis was also carried out in the BY $\mathrm{F}_{2}$ population between the SDHD G444T SNP, used for the linkage mapping procedure and the available phenotypic traits. The model used was the same as in Malek et al. (200la,b) and included effects of sex, and slaughter day. For all analyses the significance level used was $\mathrm{p}<0.05$.

\section{Results and discussion}

\section{Genotypic frequencies}

The two identified SNPs, plus the one previously described by Zhu et al. (2005), shared near complete linkage disequilibrium (LD), in 116 animals - G444 was in the same phase with T859 and G860 nucleotides, and T444 with C859 and A860. The sequence for the allele GTG was submitted to GenBank accession number DQ486897. The genotypic frequencies for both the EBVF and EBVS groups, and for the other breeds sampled (Landrace, Yorkshire, Pietrain and Duroc) are described in Table 3. The four animals that presented a different haplotype pattern from the most common ones: T444; C859; A860 or G444; T859; G860 in the growth groups were not considered for the association analysis. All animals from the other breeds presented complete LD among the three SNPs, except for the Yorkshires, where no GGTTGG genotype was observed. No significant differences were observed between the two EBV groups in relation to the number or frequency of genotypes, which was expected due to the line development and crossing strategy between the Duroc boars and commercial dams. Frequencies were also obtained for the $F_{2}$ BY pigs, but only for the G444T SNP (Table 3).

Considering the LD observed for the three SNPs, the subsequent analysis was carried out for the SDHD combined genotypes, as described in Table 3. These SNPs do not cause any amino acid change and only one (G444T) lies within the coding region of the SDHD. The other two SNPs (T859C and G860A) are located at the $3^{\prime}$-untranslated region, 378 and 377 nucleotides after the stop codon and 403 and 402 nucleotides before the PolyA signal, respectively.

\section{Linkage mapping and QTL analysis}

SDHD has been previously RH mapped to SSC9 (Zhu et al. 2005). Using the Berkshire $\times$ Yorkshire population described in Malek et al. (2001a), we conducted the linkage mapping of this gene. According to the CRIMAP analysis, SDHD maps between microsatellite markers SW827 and SW2401, at $54.6 \mathrm{cM}$, at an average distance of $2.6 \mathrm{cM}$ (LOD score of 9.19) and 2.9 cM (LOD score of 8.64), respectively, from each marker. After the QTL analysis, using the QTLEXPRESS program, the new generated map did not change significantly the QTL profile from that published by Malek et al. (200la,b). This finding was expected, as Berkshire boars and Yorkshire sows shared one of the alleles $(T)$, with its frequency for the Berkshire boars being 1.0, and for the Yorkshire sows 0.5.

No significant QTL were found when the QTLEXPRESS imprinted model was analysed, suggesting that SDHD gene does not have a parent-of-origin effect. Thomsen et al. (2004) found QTL with maternal expression for drip loss and for meat colour $(24 \mathrm{H}$ loin Hunter and $48 \mathrm{H}$ loin Minolta) in the interval SW2401-SW174 and off-flavour score in the interval SW1491-SW2401 on SSC09 using the same BY population. $S D H D$ does not map to any one of these intervals. The results obtained in our experiment suggest that another gene in SSC9, rather than $S D H D$, may have a parent-of-origin inheritance.

\section{Semiquantitative real-time PCR and correlation analysis}

There was an increase in the fold change of 1.2, between EBVF and EBVS animals $(p<0.02)$, with the $2-\Delta \mathrm{Ct}$ mean values and standard errors for EBVF and EBVS being $252.95 \pm 13.21$ and $212.06 \pm 13.06$ (arbitrary units), respectively, demonstrating that the fast-growing animals present higher mRNA levels than the pigs sired by the EBVS sires. The data were also examined for correlations between mRNA levels and phenotypic traits. The quantity of SDHD mRNA is correlated with some traits in the EBVS group $(p<0.05)$, but these correlations are not observed among the EBVF group (Table 4). When the results of both groups (EBVT) are analysed together, many traits are correlated with the normalized expression values (Table 4).

The correlations between SDHD expression and growth traits (IWT and AGE125) were highly significant for the EBVS group and when data from both groups were analysed together (EBVT). The amount of SDHD mRNA had a positive correlation to 
initial weight on test and as expected a negative correlation with age to $125 \mathrm{~kg}$ (Table 4). However, for the EBVF animals the correlation is lower but not significant. The fact that the correlation between SDHD expression levels was significant only in the EBVS, may indicate a threshold response in which once a certain level of expression is obtained further increases do not improve growth rate in these pigs. The correlations between SDHD expression and meat quality traits were also estimated (Table 4), and as for the growth traits, no significant correlation was found for the fast-growing animals. When data from all animals was combined or data from the EBVS animals were analysed, some traits were found to be correlated with SDHD expression values. It is difficult to understand how the expression of a gene as SDHD can be correlated with meat quality traits, as it is considered more like a housekeeping gene (Gottlieb \& Tomlinson 2005). The candidacy of $S D H D$ for meat quality was only accessed after the data analysis, when it was observed that not only growth, but also meat quality traits were correlated with SDHD expression values. According to our thoughts, we will only be able to access the actual effects of $S D H D$ on meat traits of pigs when the muscular biochemical pathway of $S D H D$ complex is fully understood.

The data obtained in the present experiment for the slow-growing animals, demonstrated that SDHD is positively correlated with meat temperature $2 \mathrm{~h}$ after slaughter and percentage of cooking loss of the meat. A negative correlation was found with meat juiciness. These correlations together make sense, because with higher temperature, the proteins of the meat undergo a denaturation process and consequently the meat has an increased drip loss, which reduces meat juiciness. When the data for meat quality from both groups were analysed together, the results seem to be consistent. The positively correlated traits were meat temperature $24 \mathrm{~h}$ after slaughter, firmness of the meat, $\mathrm{pH} 24 \mathrm{~h}$ and $\mathrm{pH} 120 \mathrm{~h}$ after slaughter and percentage of cooking loss. The negatively correlated traits with $S D H D$ expression are ' $a$ ' colour Minolta, moisture, juiciness and tenderness of the meat. In general, these correlations seem to be in the right direction, as with higher temperatures of the meat, we have higher water loss, less juiciness and tenderness with increased firmness, and a smaller ' $a$ ' value (redness). However, as higher $\mathrm{pH}$ should be expected to reduce drip loss and cooking loss, and more juice and tender meat, the positive correlation with the $\mathrm{pH}$ measurements does not make sense with the other trait results. In this regard, Gardner et al. (2005) working on the prediction of fresh pork quality have shown that not only $\mathrm{pH}$, but also other metabolic mechanisms, which were not assayed here, such as $\mu$ calpain autolysis and degradation of desmin are correlated with water-holding capacity and texture in pork and are at least in part independent of $\mathrm{pH}$.

\section{Association analysis of pig SDHD combined genotypes}

The normalized expression values were not affected by sex, genotype or EBV by genotype interaction $(p>0.05)$. This suggests that the causal mutation affecting $S D H D$ expression still needs to be determined. However, SDHD expression was affected by the EBV group ( $\mathrm{p}<0.02)$, as previously presented.

The growth traits, IWT and AGE125 were not affected by the genotypes $(p>0.05)$. This fact and the finding that the normalized gene expression values were highly correlated with these traits, suggest that the causative mutation associated with the expression is not the one used for genotyping. The combined genotypes were significantly associated with backfat thickness (BF), meat $\mathrm{pH}$ at $48 \mathrm{~h}$ after slaughter, moisture and off-flavour. The TGTCAG and GGTTGG animals presented a trend of higher back fat thickness. The $\mathrm{pH}$ at $48 \mathrm{~h}$ after slaughter and off-flavour score were similar for the TTCCAA and GGTTGG animals, but were higher in the TGTCAG animals (respectively, $\mathrm{p}<0.02$ and $\mathrm{p}<0.05$ ). Neither EBV nor EBV by genotype interaction affected these traits. Considering EBV by genotype interaction, GGTTGG animals in the EBVF group showed a tendency for higher moisture than the other EBV by genotype combinations (Table 5).

Association analysis for the BY population is presented in Table 6. As in all other breeds there was near complete LD between the three SNPs, and only G444T was genotyped in the BY population. Despite the fact that $S D H D$ did not change the QTL profile on SSC09, its G444T genotype is associated with more traits in BY population than in the growth groups. It should be remembered that the EBV groups had small number of animals each, which is a different situation for the BY population as it has approximately 500 animals. However, it should be noted that the $\mathrm{BY}$ population are $\mathrm{F}_{2}$ animals and this probably created LD. In this kind of population, a mutation in a candidate gene, as the one described here may be only a marker, instead of being the causative mutation for the associated phenotypes. In the BY population, the T444 allele (TCA allele), in heterozygous or in homozygous condition, seems to be the most favourable one, as the animals carry- 
ing it had the desirable phenotypes, such as a higher average daily gain, less cholesterol, reduced back fat at the last rib, reduced drip and cooking loss, better water-holding capacity, higher $\mathrm{pH}$ and darker colour of the meat.

The G444T nucleotide substitution additive and dominance effects are also shown in Table 4. The allele T444 had additive favourable effects increasing the values for almost all traits, like average daily gain, colour, different measurements of $\mathrm{pH}$, juiciness and tenderness of the meat. The GG genotype additive effects were in general associated with the less valuable phenotypic traits. For the dominance effects, the same pattern described above was observed, with the $T$ carrying genotypes having the most favourable values for each trait.

In this study, we have describe not only the genotype effects of $S D H D$ gene on production traits in pigs, but also the effects of its expression, evaluated by semiquantitative real-time PCR. One of the most interesting findings is the correlation between $S D H D$ expression values and growth. However, it still needs to be determined if this effect is a cause for or a consequence of the body development in pigs. On the other hand, the association of this gene with meat quality traits, not only for the effects of the expression levels, but also for the genotype effects, suggests that it may play an important role in glycolysis. The results obtained here were consistent and the semiquantitative real-time PCR seems to be a technique that could be used in the identification of candidate genes in animal production.

Further investigation into the importance of $S D H D$ in animal production and its effects on growth and meat quality is needed. Although the described SNPs in this experiment were not significantly associated with expression levels, expression levels of $S D H D$ were highly associated with both growth and meat quality traits in these animals. It is possible that these SNPs do impact the role of SDHD independently of expression levels.

The associations between the quantity of SDHD mRNA in muscle tissue and growth performance and meat quality traits in pigs should encourage more research to understand better the role of $S D H D$ on pig physiology and its relation to growth and meat traits. Further work to examine the importance of the expression level of this candidate gene in both muscle and liver tissue, not just in pigs, but also in other livestock species, could provide novel strategies for improving meat quality and the efficiency of animal production.

\section{Acknowledgements}

Financial help provided from Brazil by CNPq for providing support to Simone Guimaraes and the National Pork Board, the Iowa Agriculture and Home Economics Experiment Station, Hatch and State of Iowa Funds and PIC USA is appreciated. The authors thank Kimberly Glenn and Mei Yu for the technical assistance. Assistance provided by Alan Mileham for the pyrosequencing is also appreciated.

\section{References}

Gardner M.A., Huff-Lonergan E., Lonergan S.M. (2005) Prediction of Fresh Pork Quality using Indicators of Protein Degradation and Calpain Activation. Proceedings of the 51st International Congress of Meat Science and Technology, 7-12 August. American Meat Science Association, Baltimore, Maryland, USA.

Gottlieb E., Tomlinson I.P.M. (2005) Mitochondrial tumour suppressors: a genetic and biochemical update. Nat. Rev. Cancer, 5, 857-866.

Green P., Falls K., Crooks S. (1990) Documentation for CRIMAP, version 2.4. Washington Univ. School of Medicine, St Louis, MO.

Khatib H. (2005) The COPG2, DCN and SDHD genes are biallelically expressed in cattle. Mamm. Genome, 16, 545-552.

Livak K.J., Schmittgen T.D. (2001) Analysis of relative gene expression data using real-time quantitative PCR and the 2- $\Delta \Delta \mathrm{CT}$ method. Methods, 25, 402-408.

Malek M., Dekkers J.C.M., Lee H.K., Baas T.J., Rothschild M.F. (2001a) A molecular genome scan analysis to identify chromosomal regions influencing economic traits in the pig: I. Growth and body composition. Mamm. Genome, 12, 630-636.

Malek M., Dekkers J.C.M., Lee H.K., Baas T.J., Prusa K., Huff-Lonergan E, Rothschild M.F. (2001b) A molecular genome scan analysis to identify chromosomal regions influencing economic traits in the pig: II. Meat and muscle composition. Mamm. Genome, 12, 637-642.

Seaton G., Haley C.S., Knott S.A., Kearsey M., Visscher P.M. (2002) QTL Express: mapping quantitative trait loci in simple and complex traits. Bioinformatics, 18, 339-340.

Thomsen H., Lee H.K., Rothschild M.F., Malek M., Dekkers J.C.M. (2004) Characterization of quantitative trait loci for growth and meat quality in a cross between commercial breeds of swine. J. Anim. Sci., 82, 2213-2228.

Zhu Z.M., Zhang J.B., Zhao S.H. (2005) Cloning, mapping and association study with carcass traits of the porcine SDHD gene. Anim. Genet., 36, 191-195. 